\title{
ANALYTIC NON-INTEGRABILITY OF THE SUSLOV PROBLEM
}

\author{
ADAM MAHDI ${ }^{1}$ AND CLAUDIA VALLS ${ }^{2}$
}

\begin{abstract}
In this work we consider the Suslov problem, which consists of a rotation motion of a rigid body, whose center of mass is located at one axis of inertia, around a fixed point $O$ in a constant gravity field restricted to a nonholonomic constraint. The integrability and non-integrability has been established by a number of authors for the nongeneric values of $\mathbf{b}=\left(b_{1}, b_{2}, b_{3}\right)$ which is the unit vector along the line connecting the point $O$ with the center of mass of the body. Here we prove the analytic non-integrability for the remaining (generic) values of $\mathbf{b}$.
\end{abstract}

\section{INTRODUCTION}

The Suslov problem is one of the most famous problems in nonholonomic dynamics with no shape space and was formulated in [6]. It is a generalized rigid body with some of its body angular velocity components set equal to zero, i.e., it consists of a rotational motion of a rigid body around a fixed point $O$ in a constant gravity field when restricted by a non-holonomic constraint

$$
\langle\mathbf{n}, \boldsymbol{\omega}\rangle=0,
$$

where $\boldsymbol{\omega}=\left(\omega_{1}, \omega_{2}, \omega_{3}\right)$ is the body angular velocity, $\mathbf{n}$ is a vector fixed in the body and $\langle\cdot, \cdot\rangle$ denotes the standard metric in $\mathbb{R}^{3}$. To be more precise, the equations of motion of the Suslov problem are

$$
\mathbf{I} \dot{\omega}=\mathbf{I} \boldsymbol{\omega} \times \boldsymbol{\omega}+\varepsilon \boldsymbol{\gamma} \times \mathbf{b}+\lambda \mathbf{n}, \quad \dot{\boldsymbol{\gamma}}=\boldsymbol{\gamma} \times \boldsymbol{\omega}, \quad\langle\mathbf{n}, \boldsymbol{\omega}\rangle=0,
$$

where $\lambda$ is the Langrange multiplier; the diagonal matrix $\mathbf{I}=\operatorname{diag}\left(I_{1}, I_{2}, I_{3}\right)$ represents the inertia of the body; $\gamma=\left(\gamma_{1}, \gamma_{2}, \gamma_{3}\right)$ is the unit vertical vector and $\mathbf{b}=\left(b_{1}, b_{2}, b_{3}\right)$ is the unit vector along the line connecting the point $O$ with the center of mass of the body; $\varepsilon$ is the product of the mass of the body and the gravity constant. We assume that $\mathbf{n}=(0,0,1)$, i.e., we assume that the center of mass is located at the third axis of inertia, and thus the equation of the constraint is $\omega_{3}=0$. The Suslov equations can be written as

$$
\begin{gathered}
\dot{\omega}_{1}=\frac{\varepsilon}{I_{1}}\left(\gamma_{2} b_{3}-\gamma_{3} b_{2}\right), \quad \dot{\omega}_{2}=\frac{\varepsilon}{I_{2}}\left(\gamma_{3} b_{1}-\gamma_{1} b_{3}\right), \\
\dot{\gamma}_{1}=-\omega_{2} \gamma_{3}, \quad \dot{\gamma}_{2}=\omega_{1} \gamma_{3}, \quad \dot{\gamma}_{3}=\omega_{2} \gamma_{1}-\omega_{1} \gamma_{2},
\end{gathered}
$$

where $I_{1}, I_{2}>0$. System (2) has two polynomial first integrals

$$
\mathcal{F}_{1}=\frac{1}{2}\left(I_{1} \omega_{1}^{2}+I_{2} \omega_{2}^{2}\right)+\varepsilon\left(b_{1} \gamma_{1}+b_{2} \gamma_{2}+b_{3} \gamma_{3}\right) \quad \text { and } \quad \mathcal{F}_{2}=\gamma_{1}^{2}+\gamma_{2}^{2}+\gamma_{3}^{2} .
$$

The integrability of the Suslov problem and its generalization have been studied by a number of authors (see [3], [4], [5], [1], [8], [7] and the references therein). There are three known integrable cases of system (2), which are:

(i) The Suslov case (see [6]), i.e., when $\varepsilon=0$. Then $\mathcal{F}_{3}=\omega_{1}$. This case was studied by Suslov in [6] without the assumption on the location of the center of mass, i.e., with $\mathbf{n}$ being arbitrary.

(ii) The Kharlamova-Zabelina case (see [2]), i.e., when $\langle\mathbf{b}, \mathbf{n}\rangle=0$. Then $\mathcal{F}_{3}=I_{1} \omega_{1} b_{1}+$ $I_{2} \omega_{2} b_{2}$.

2010 Mathematics Subject Classification. 70F25, 70E40, 37J30.

Key words and phrases. Suslov problem, analytic integrability.

The second author is supported by the grants AGAUR PIV-DGR-2010 and by FCT through CAMGDS, Lisbon. 
(iii) The Kozlov case, i.e., when $\mathbf{b}$ parallel to $\mathbf{n}$, i.e., $b_{1}=b_{2}=0$, and $I_{1}=I_{2}$. Then $\mathcal{F}_{3}=\omega_{1} \gamma_{1}+\omega_{2} \gamma_{2}($ see $[3])$.

There are also a number of non-integrability results. Namely, using different type of techniques such as the Moralis-Ramis theory, or the study of the connection between the properties of solutions on the complex time plane and the existence of first integrals it was proved in $[8,7]$ and [4] that when $\mathbf{b}$ is parallel to $\mathbf{n}$ only the case $I_{1}=I_{2}$ admits an additional complex or real meromorphic first integral. When $\mathbf{b}$ is not parallel to $\mathbf{n}$ and $b_{2}=0$ it was proved in [5] and [4] that system (2) does not possess any third complex or real meromorphic first integral which is functionally independent of $\mathcal{F}_{1}$ and $\mathcal{F}_{2}$. The main purpose of this paper is to study the integrability of the remaining case, i.e., when $\varepsilon b_{2} b_{3} \neq 0$. We note that if $\varepsilon \neq 0$, then with a rescaling of time we can always assume that $\varepsilon=1$. Moreover, we note that system (2) is invariant with respect to the change of variables

$$
\begin{aligned}
& \gamma_{1} \rightarrow-\gamma_{2}, \quad \gamma_{2} \rightarrow-\gamma_{1}, \quad \omega_{1} \rightarrow \omega_{2}, \quad \omega_{2} \rightarrow \omega_{1}, \\
& I_{2} \rightarrow I_{1}, \quad I_{1} \rightarrow I_{2}, \quad b_{2} \rightarrow-b_{1}, \quad b_{1} \rightarrow-b_{2} .
\end{aligned}
$$

Therefore we can also assume that $b_{1} \neq 0$ otherwise with the above change we are in the case $b_{2}=0$, which was studied in $[8,7]$ and [4]. In short, we will consider the polynomial integrability of system $(2)$ when $\varepsilon b_{1} b_{2} b_{3} \neq 0$. The following theorem is the main result of the paper.

Theorem 1. If $\varepsilon b_{1} b_{2} b_{3} \neq 0$, then system (2) does not admit any third analytic first integral which is functionally independent of $\mathcal{F}_{1}$ and $\mathcal{F}_{2}$.

This theorem completes the characterization of the integrability of the Suslov problem in the analytic category. The article is organised as follows. In Section 2 we shortly review some of the definitions and results used in the paper. The proof of our main result is given in Section 3

\section{Preliminaries}

We consider polynomial differential systems of the form

$$
\frac{d \mathbf{x}}{d t}=\dot{\mathbf{x}}=\mathbf{P}(\mathbf{x}), \quad \mathbf{x}=\left(x_{1}, \ldots, x_{n}\right) \in \mathbb{C}^{n},
$$

with $\mathbf{P}(\mathbf{x})=\left(P_{1}(\mathbf{x}), \ldots, P_{n}(\mathbf{x})\right)$ and $P_{i} \in \mathbb{C}\left[x_{1}, \ldots, x_{n}\right]$ for $i=1, \ldots, n$. As usual $\mathbb{C}\left[x_{1}, \ldots, x_{n}\right]$ denotes the polynomial right over $\mathbb{C}$ in the variables $x_{1}, \ldots, x_{n}$. Here, $t$ can be real or complex. We say that $\mathcal{F}$ is a first integral of (4) if and only if $\mathcal{F}$ is constant on the solution curves of (4). We associate to (4) a vector field $X$ as follows

$$
X=P_{1}(\mathbf{x}) \frac{\partial}{\partial x_{1}}+\ldots+P_{n}(\mathbf{x}) \frac{\partial}{\partial x_{n}} .
$$

Then if $\mathcal{F}$ is differentiable, then it is a first integral of (4) if and only if $X \mathcal{F}=0$. We say that $\mathcal{F}$ is a polynomial, analytic or meromorphic first integral depending whether $\mathcal{F}$ belongs to the corresponding category of functions. Also, we say that system (4) is weighthomogeneous if there exists $\mathbf{s}=\left(s_{1}, \ldots, s_{n}\right) \in \mathbb{N}^{n}$ and $d \in \mathbb{N}$ such that for an arbitrary $\alpha \in \mathbb{R}^{+}$, where $\mathbb{R}^{+}$denote the set of positive real numbers, we have

$$
P_{i}\left(\alpha^{s_{1}} x_{1}, \ldots, \alpha^{s_{n}} x_{n}\right)=\alpha^{s_{i}-1+d} P_{i}\left(x_{1}, \ldots, x_{n}\right)
$$

for $i=1, \ldots, n$. We call $\mathbf{s}=\left(s_{1}, \ldots, s_{n}\right)$ the weight-exponent of system (4) and $d$ the weight degree with respect to the weight exponent $\mathbf{s}$. In the particular case that $\mathbf{s}=(1, \ldots, 1)$ system (4) is called a homogeneous polynomial differential system of degree $d$. We say that a polynomial $F\left(x_{1}, \ldots, x_{n}\right)$ is a weight-homogeneous polynomial with weight exponent $\mathbf{s}=\left(s_{1}, \ldots, s_{5}\right)$ and weight-degree $d$ if

$$
F\left(\alpha^{s_{1}} x_{1}, \ldots, \alpha^{s_{n}} x_{n}\right)=\alpha^{d} F\left(x_{1}, \ldots, x_{n}\right) .
$$

If $\mathbf{s}=(1, \ldots, 1)$ then we say that $F$ is a homogeneous polynomial of degree $d$. The following well-known proposition reduces the study of the existence of an analytic first integral of a 
weight-homogeneous polynomial differential system (4) to the study of the existence of a weight-homogeneous polynomial first integrals.

Proposition 1. Let $H$ be an analytic function and let $H=\sum_{k} H_{k}$ be its decomposition into weight-homogeneous polynomials of weight degree $k$ with respect to the weight exponent s. Then $H$ is an analytic first integral of the weight-homogeneous polynomial differential system (4) with weight exponent $\mathbf{s}$ if and only if each weight-homogeneous part $H_{k}$ is a first integral of system (4) for all $k$.

Introducing the change of variables

$$
\Omega_{1}=\ell_{1} \omega_{1}+i \ell_{2} \omega_{2}, \quad \Omega_{2}=\ell_{1} \omega_{1}-i \ell_{2} \omega_{2}, \quad \xi_{1}=\gamma_{1}+i \gamma_{3}, \quad \xi_{3}=\gamma_{1}-i \gamma_{3},
$$

where $i=\sqrt{-1}$ and $\ell_{1}=\sqrt{I_{1}}$ and $\ell_{2}=\sqrt{I_{2}}$, system (2) writes

$$
\begin{aligned}
& \dot{\Omega}_{1}=-\frac{i}{2 \ell_{2}}\left(b_{3}\left(\xi_{1}+\xi_{3}\right)+i b_{1}\left(\xi_{1}-\xi_{3}\right)\right)+\frac{1}{2 \ell_{1}}\left(2 b_{3} \gamma_{2}+i b_{2}\left(\xi_{1}-\xi_{3}\right)\right), \\
& \dot{\Omega}_{2}=\frac{i}{2 \ell_{2}}\left(b_{3}\left(\xi_{1}+\xi_{3}\right)+i b_{1}\left(\xi_{1}-\xi_{3}\right)\right)+\frac{1}{2 \ell_{1}}\left(2 b_{3} \gamma_{2}+i b_{2}\left(\xi_{1}-\xi_{3}\right)\right), \\
& \dot{\xi}_{1}=\frac{\xi_{1}}{2 \ell_{2}}\left(\Omega_{1}-\Omega_{2}\right)-\frac{i \gamma_{2}}{2 \ell_{1}}\left(\Omega_{1}+\Omega_{2}\right), \\
& \dot{\gamma}_{2}=-\frac{i}{4 \ell_{1}}\left(\xi_{1}-\xi_{3}\right)\left(\Omega_{1}+\Omega_{2}\right), \\
& \dot{\xi}_{3}=-\frac{\xi_{3}}{2 \ell_{1}}\left(\Omega_{1}-\Omega_{2}\right)+\frac{i \gamma_{2}}{2 \ell_{2}}\left(\Omega_{1}+\Omega_{2}\right) .
\end{aligned}
$$

The first integral $\mathcal{F}_{1}$ in these new variables becomes

$$
\overline{\mathcal{F}}_{1}=\mathcal{F}_{1}\left(\gamma_{1}, \gamma_{2}, \gamma_{3}, \Omega_{1}, \Omega_{2}\right)=\Omega_{1} \Omega_{2}+b_{1}\left(\xi_{1}+\xi_{3}\right)+2 b_{2} \gamma_{2}-i b_{3}\left(\xi_{1}-\xi_{3}\right) .
$$

The second integral $\mathcal{F}_{2}$ in these new variables becomes

$$
\overline{\mathcal{F}}_{2}=\mathcal{F}_{2}\left(\gamma_{1}, \gamma_{2}, \gamma_{3}, \Omega_{1}, \Omega_{2}\right)=\xi_{1} \xi_{3}+\gamma_{2}^{2} .
$$

System (5) is weight-homogeneous of weight exponent $(1,1,2,2,2)$ and weight degree $d=2$. By Proposition 1 the characterization of the polynomial first integral of weight exponent $(1,1,2,2,2)$ and weight degree $d=2$ of system (5) is sufficient to know all the analytic first integrals of system (5). Thus for each $n \geq 1$, we can express any analytic first integrals $G$ as

$$
G=\sum_{l_{1}+l_{2}+2 j_{1}+2 j_{2}+2 j_{3}=n} G_{l_{1}, l_{2}, j_{1}, j_{2}, j_{3}} \Omega_{1}^{l_{1}} \Omega_{2}^{l_{2}} \xi_{1}^{j_{1}} \gamma_{2}^{j_{2}} \xi_{3}^{j_{3}},
$$

where $G_{l_{1}, l_{2}, j_{1}, j_{2}, j_{3}} \in \mathbb{C}$. In the rest of this section we shall use that fact that the five dimensional system (5) possesses the two functionally independent polynomial first integrals $\overline{\mathcal{F}}_{1}$ and $\overline{\mathcal{F}}_{2}$ in order to reduce it to the three dimensional polynomial system. We shall restrict system (5) to the zero level of the first integral $\overline{\mathcal{F}}_{2}$. Hence, setting $\overline{\mathcal{F}}_{2}=0$ and solving for $\xi_{3}$ we get

$$
\xi_{3}=-\frac{\gamma_{2}^{2}}{\xi_{1}} .
$$

Now introducing $\xi_{3}=-\gamma_{2}^{2} / \xi_{1}$ into $\overline{\mathcal{F}}_{1}=0$ sand solving for $\Omega_{1}$ we get

$$
\Omega_{1}=-\frac{1}{\Omega_{2} \xi_{1}}\left(b_{1}\left(\xi_{1}^{2}-\gamma_{2}^{2}\right)+2 b_{2} \gamma_{2} \xi_{1}-i b_{3}\left(\xi_{1}^{2}+\gamma_{2}^{2}\right) .\right.
$$

Let

$$
\begin{aligned}
\mathcal{S} & =i b_{1}\left(\xi_{1}^{2}-\gamma_{2}^{2}\right)+b_{3}\left(\xi_{1}^{2}+\gamma_{2}^{2}\right)+2 i b_{2} \xi_{1} \gamma_{2} \\
\mathcal{S}_{1} & =\left(i b_{2} \ell_{2}-b_{1} \ell_{1}\right)\left(\xi_{1}^{2}+\gamma_{2}^{2}\right)+b_{3}\left(2 \ell_{2} \xi_{1} \gamma_{2}+i \ell_{1}\left(\xi_{1}^{2}-\gamma_{2}^{2}\right)\right) .
\end{aligned}
$$


Then system (5) with the restriction $\overline{\mathcal{F}}_{1}=\overline{\mathcal{F}}_{2}=0$ and with a rescaling of time by setting $d t=\ell_{1} \ell_{2} \Omega_{2} \xi_{1} d \tau$ becomes

$$
\begin{aligned}
\dot{\Omega}_{2} & =2 \Omega_{2} \xi_{1} \mathcal{S}_{1}, \\
\dot{\xi}_{1} & =2 \xi_{1}\left(i\left(\ell_{1} \xi_{1}-i \ell_{2} \gamma_{2}\right) \mathcal{S}-\xi_{1}\left(\ell_{1} \xi_{1}+i \ell_{2} \gamma_{2}\right) \Omega_{2}^{2}\right), \\
\dot{\gamma}_{2} & =\ell_{2}\left(\xi_{1}^{2}+\gamma_{2}^{2}\right)\left(\mathcal{S}-i \xi_{1} \Omega_{2}^{2}\right) .
\end{aligned}
$$

where the dot denotes derivative with respect to $\tau$. Then if we denote by $\bar{G}=\bar{G}\left(\Omega_{2}, \xi_{1}, \gamma_{2}\right)$ the restriction of $G$ given in (6) to $\xi_{3}$ and $\Omega_{1}$ as in (7) and (8) we get that $\bar{G}$ is a first integral of system (10) and is of the form

(11)

$\bar{G}=\sum_{l_{1}+l_{2}+2 j_{1}+2 j_{2}+2 j_{3}=n} \bar{G}_{1,2,3} \Omega_{2}^{l_{2}-l_{1}}\left(b_{1}\left(\xi_{1}^{2}-\gamma_{2}^{2}\right)+2 b_{2} \gamma_{2} \xi_{1}-i b_{3}\left(\xi_{1}^{2}+\gamma_{2}^{2}\right)\right)^{l_{1}} \xi_{1}^{j_{1}-j_{3}-l_{1}} \gamma_{2}^{j_{2}+2 j_{3}}$,

where $\bar{G}_{1,2,3}=(-1)^{l_{1}+j_{3}} G_{l_{1}, l_{2}, j_{1}, j_{2}, j_{3}} \in \mathbb{C}$.

\section{Proof of the main Result}

It follows from Section 2 that to prove Theorem 1 it is sufficient to show that system (10) has no first integral of the form (11). For each $n \geq 1$, we can express it as

$$
G=\sum_{k=-[n / 2]}^{[n / 2]} G_{k}\left(\xi_{1}, \gamma_{2}\right) \Omega_{2}^{k}
$$

where as usual [.] denotes the integer part function. Without loss of generality we can assume that $G$ has no constant term. We note that $G_{k}$ is a polynomial in the variable $\gamma_{2}$ and a rational function with respect to the variable $\xi_{1}$. Clearly, $G$ is a polynomial in the variable $\gamma_{2}$ and a rational function in the variables $\xi_{1}$ and $\Omega_{2}$. To prove Theorem 1 it is enough to show that $G_{k}=0$ for each $k=-[n / 2], \ldots,[n / 2]$. If $G$ is a first integral system (10) it must satisfy

$$
\frac{\partial G}{\partial \Omega_{2}} \dot{\Omega}_{2}+\frac{\partial G}{\partial \xi_{1}} \dot{\xi}_{1}+\frac{\partial G}{\partial \gamma_{2}} \dot{\gamma}_{2}=0
$$

Setting

$$
\mathcal{A}\left[f_{j}\right]=\mathcal{S}\left[\ell_{2}\left(\xi_{1}^{2}+\gamma_{2}^{2}\right) \frac{\partial f_{j}}{\partial \gamma_{2}}+2 i \xi_{1}\left(\ell_{1} \xi_{1}-i \ell_{2} \gamma_{2}\right) \frac{\partial f_{j}}{\partial \xi_{1}}\right]+2 j \xi_{1} \mathcal{S}_{1} f_{j}
$$

and

$$
\mathcal{B}\left[f_{j}\right]=-i \ell_{2}\left(\xi_{1}^{2}+\gamma_{2}^{2}\right) \xi_{1} \frac{\partial f_{j}}{\partial \gamma_{2}}-2 \xi_{1}^{2}\left(\ell_{1} \xi_{1}+i \ell_{2} \gamma_{2}\right) \frac{\partial f_{j}}{\partial \xi_{1}},
$$

computing the different coefficients of $\Omega_{2}^{j}$ in (12), we obtain

$$
\begin{aligned}
\mathcal{A}\left[G_{j}\right] & =0, & & j=-n,-n+1, \\
\mathcal{A}\left[G_{j}\right]+\mathcal{B}\left[G_{j-2}\right] & =0, & & j=-n+2, \ldots, n, \\
\mathcal{B}\left[G_{j}\right] & =0, & & j=n-1, n .
\end{aligned}
$$

We consider a preliminary result.

Lemma 2. Let $\mathcal{S}$ and $\mathcal{S}_{1}$ be as in (9), then $\mathcal{S}$ does not divide $\mathcal{S}_{1}$.

Proof. Setting $\mathcal{S}=0$ and solving with respect to $\xi_{1}$ we get

$$
\xi_{1}=\frac{\gamma_{2}}{b_{1}-i b_{3}}\left(-b_{2} \pm \sqrt{b_{1}^{2}+b_{2}^{2}+b_{3}^{3}}\right) .
$$

Note that since $b_{1}, b_{3} \in \mathbb{R}, \xi_{1}$ is well defined. Now introducing $\xi_{1}$ in $\mathcal{S}_{1}$ we obtain

$$
\begin{aligned}
& \frac{2 \gamma_{2}^{2}}{\left(b_{1}-i b_{3}\right)^{2}} \sqrt{b_{1}^{2}+b_{2}^{2}+b_{3}^{2}}\left(b_{2} \ell_{1}\left(b_{3}+i b_{1}\right)+\ell_{2}\left(b_{2}^{2}+i b_{1} b_{3}+b_{3}^{2}\right)\right. \\
& \left.+\sqrt{b_{1}^{2}+b_{2}^{2}+b_{3}^{2}}\left(i b_{2} \ell_{2}-\left(b_{1}-i b_{3}\right) \ell_{1}\right)\right) .
\end{aligned}
$$


Solving it with respect to $b_{1}$ we get

$$
b_{1}= \pm i \sqrt{b_{2}^{2}+b_{3}^{2}} \quad \text { and } \quad b_{1}=\frac{1}{I_{1}}\left[i b_{2} \sqrt{I_{1} I_{2}} \pm b_{3} \sqrt{I_{1}\left(I_{2}-I_{1}\right)}\right]
$$

which is not possible since $b_{j} \in \mathbb{R}$ for $j=1,2,3$ and $I_{1}, I_{2}>0$.

Lemma 3. Let $l_{1} \neq l_{2}$ and $f_{j}$ be a polynomial in the variable $\gamma_{2}$ and a rational function with respect to the variable $\xi_{1}$. Then condition $\mathcal{A}\left[f_{j}\right]=0$ implies

$$
f_{j}= \begin{cases}0, & \text { for } \quad j \in \mathbb{Z} \backslash\{0\}, \\ \text { constant, } & \text { for } j=0 .\end{cases}
$$

Proof. Let $l_{1} \neq l_{2}$ and $j \in \mathbb{Z} \backslash\{0\}$. From $\mathcal{A}\left[f_{j}\right]=0$ we have that

$$
\mathcal{S}\left[\ell_{2}\left(\xi_{1}^{2}+\gamma_{2}^{2}\right) \frac{\partial f_{j}}{\partial \gamma_{2}}+2 i \xi_{1}\left(\ell_{1} \xi_{1}-i \ell_{2} \gamma_{2}\right) \frac{\partial f_{j}}{\partial \xi_{1}}\right]+2 j \xi_{1} \mathcal{S}_{1} f_{j}=0 .
$$

Since by Lemma $2 \mathcal{S}$ does not divide $\mathcal{S}_{1}$, in view of $(15)$ we get that $\mathcal{S}$ must divide $f_{j}$. Therefore we can write $f_{j}=\mathcal{S}^{m} g_{j}$ for some $m \geq 1$ and $g_{j}$ is not divisible by $\mathcal{S}$. Then $g_{j}$ satisfies

$$
m \mathcal{S}\left[\ell_{2}\left(\xi_{1}^{2}+\gamma_{2}^{2}\right) \frac{\partial g_{j}}{\partial \gamma_{2}}+2 i \xi_{1}\left(\ell_{1} \xi_{1}-i \ell_{2} \gamma_{2}\right) \frac{\partial g_{j}}{\partial \xi_{1}}\right]+G_{j} g_{j}=0
$$

where

$$
\begin{aligned}
G_{j}= & -2\left(b_{1} j l_{1} \xi_{1}^{3}+b_{1} j l_{1} \xi_{1} \gamma_{2}^{2}-2 b_{1} m l_{1} \xi_{1}^{2} \gamma_{2}+i b_{1} m l_{2} \xi_{1}^{2} \gamma_{2}+2 i b_{1} m l_{2} \xi_{1} \gamma_{2}^{2}+i b_{1} m l_{2} \gamma_{2}^{3}-i b_{2} j l_{2} \xi_{1}^{3}\right. \\
& -i b_{2} j m_{2} \xi_{1} \gamma_{2}^{2}+2 b_{2} m l_{1} \xi_{1}^{3}-i b_{2} m l_{2} \xi_{1}^{3}-2 i b_{2} l l_{2} \xi_{1}^{2} \gamma_{2}-i b_{2} m l_{2} \xi_{1} \gamma_{2}^{2}-i b_{3} j l_{1} \xi_{1}^{3}+i b_{3} j l_{1} \xi_{1} \gamma_{2}^{2} \\
& \left.-2 b_{3} j l_{2} \xi_{1}^{2} \gamma_{2}-2 i b_{3} m l_{1} \xi_{1}^{2} \gamma_{2}-b_{3} m l_{2} \xi_{1}^{2} \gamma_{2}-2 b_{3} m l_{2} \xi_{1} \gamma_{2}^{2}-b_{3} m l_{2} \gamma_{2}^{3}\right) .
\end{aligned}
$$

Since $g_{j}$ is not divisible by $\mathcal{S}$, then $G_{j}$ must be divisible by $\mathcal{S}$, i.e.

$$
G_{j}=\mathcal{S}\left(\alpha_{1} \xi_{1}+\alpha_{2} \gamma_{2}+\alpha_{3}\right),
$$

for some $\alpha_{i} \in \mathbb{C}, i=1,2,3$. Then introducing $G_{j}$ in (16), and after simplifying by $\mathcal{S}$, we get

$$
m\left[\ell_{2}\left(\xi_{1}^{2}+\gamma_{2}^{2}\right) \frac{\partial g_{j}}{\partial \gamma_{2}}+2 i \xi_{1}\left(\ell_{1} \xi_{1}-i \ell_{2} \gamma_{2}\right) \frac{\partial g_{j}}{\partial \xi_{1}}\right]+\left(\alpha_{1} \xi_{1}+\alpha_{2} \gamma_{2}+\alpha_{3}\right) g_{j}=0
$$

Solving it we obtain

$$
g_{j}=K_{j}\left(\frac{l_{2}^{2} \xi_{1}^{2}-2 i l_{1} l_{2} \xi_{1} \gamma_{2}-l_{2}^{2} \gamma_{2}^{2}}{\xi_{1}}\right) e^{-\frac{\xi_{1} h\left(\xi_{1}, \gamma_{2}\right)}{2 m \sqrt{l_{1}^{2}-l_{2}^{2}\left(l_{2}^{2} \xi_{1}^{2}-2 i l_{1} l_{2} \xi_{1} \gamma_{2}-l_{2}^{2} \gamma_{2}^{2}\right)}}},
$$

where $K_{j}$ is any smooth function in the variable $\left(l_{2}^{2} \xi_{1}^{2}-2 i l_{1} l_{2} \xi_{1} \gamma_{2}-l_{2}^{2} \gamma_{2}^{2}\right) / \xi_{1}$ and

$$
\begin{aligned}
h_{1}= & -2 \alpha_{3} \sqrt{l_{1}^{2}-l_{2}^{2}}\left(l_{1} \xi_{1}-i l_{2} \gamma_{2}\right)-\left(l_{2}\left(\xi_{1}^{2}-\gamma_{2}^{2}\right)-2 i l_{1} \xi_{1} \gamma_{2}\right)\left(i \alpha_{2} \sqrt{l_{1}^{2}-l_{2}^{2}} \log \alpha_{1}\right. \\
& \left.-2 i\left(\alpha_{2} l_{1}+i \alpha_{1} l_{2}\right) \log \left(\frac{-2 i \sqrt{l_{1}^{2}-l_{2}^{2}} \xi_{1}-2 i\left(l_{1} \xi_{1}-i l_{2} \gamma_{2}\right)}{\sqrt{x_{1}}}\right)\right) .
\end{aligned}
$$

Since $m \geq 1, l_{1} \neq l_{2}, l_{1} l_{2} \neq 0$ and $g_{j}$ must be a polynomial in the variable $\gamma_{2}$ and a rational function with respect to the variable $\xi_{1}$, we must have $\alpha_{1}=\alpha_{2}=\alpha_{3}=0$. But then $G_{j}=0$, which is not possible. Hence, $g_{j}=0$ which yields $f_{j}=0$. Now assume $j=0$. From $\mathcal{A}\left[f_{0}\right]=0$ after simplifying by $\mathcal{S}$, we have that

$$
\ell_{2}\left(\xi_{1}^{2}+\gamma_{2}^{2}\right) \frac{\partial f_{0}}{\partial \gamma_{2}}+2 i \xi_{1}\left(\ell_{1} \xi_{1}-i \ell_{2} \gamma_{2}\right) \frac{\partial f_{0}}{\partial \xi_{1}}=0
$$

Solving it we obtain

$$
f_{0}=K_{0}\left(\frac{l_{2}^{2} \xi_{1}^{2}-2 i l_{1} l_{2} \xi_{1} \gamma_{2}-l_{2}^{2} \gamma_{2}^{2}}{\xi_{1}}\right)
$$


where $K_{j}$ is any smooth function. Since $f_{0}$ must be a polynomial in the variable $\gamma_{2}$ and a Laurent polynomial with respect to the variable $\xi$ we must have that $K_{0}$ is a polynomial. In view of equation (11), we get that $f_{0}$ is of the form

(19) $f_{0}=\sum_{2 l_{2}+2 j_{1}+2 j_{2}+2 j_{3}=n} \bar{G}_{1,2,3}\left(b_{1}\left(\xi_{1}^{2}-\gamma_{2}^{2}\right)+2 b_{2} \gamma_{2} \xi_{1}-i b_{3}\left(\xi_{1}^{2}+\gamma_{2}^{2}\right)\right)^{l_{2}} \xi_{1}^{j_{1}-j_{3}-l_{2}} \gamma_{2}^{j_{2}+2 j_{3}}$.

Then $n$ must even, and comparing (18) and (19) if we set $j_{3}+l_{2}-j_{1}=p$, we must have $2 l_{2}+j_{2}+2 j_{3}=2 p$. This implies, $2 j_{3}+2 l_{2}-2 j_{1}=2 l_{2}+j_{2}+2 j_{3}$ which yields $-2 j_{1}=j_{2}$, i.e. $j_{1}=j_{2}=0$. Hence, if we denote $G_{l_{2}, l_{2}, 0,0, j_{3}}=\tilde{G}_{l_{2}, j_{3}}$ we get

$$
\begin{aligned}
f_{0} & =\sum_{2 l_{2}+2 j_{3}=n}(-1)^{j_{3}+l_{2}} \tilde{G}_{l_{2}, j_{3}}\left(b_{1}\left(\xi_{1}^{2}-\gamma_{2}^{2}\right)+2 b_{2} \gamma_{2} \xi_{1}-i b_{3}\left(\xi_{1}^{2}+\gamma_{2}^{2}\right)\right)^{l_{2}} \xi_{1}^{-j_{3}-l_{2}} \gamma_{2}^{2 j_{3}} \\
& =\frac{(-1)^{n / 2}}{\xi_{1}^{n / 2}} \sum_{l_{2}=0}^{n / 2} \tilde{G}_{l_{2}, \frac{n-2 l_{2}}{2}}\left(b_{1}\left(\xi_{1}^{2}-\gamma_{2}^{2}\right)+2 b_{2} \gamma_{2} \xi_{1}-i b_{3}\left(\xi_{1}^{2}+\gamma_{2}^{2}\right)\right)^{l_{2}} \gamma_{2}^{n-2 l_{2}} .
\end{aligned}
$$

Comparing with (18) we obtain that if $f_{0}$ is not a constant then

$\left(l_{2}^{2} \xi_{1}^{2}-2 i l_{1} l_{2} \xi_{1} \gamma_{2}-l_{2}^{2} \gamma_{2}^{2}\right)^{n / 2}=\sum_{l_{2}=0}^{n / 2} \bar{G}_{l_{2}, l_{2}, \frac{n-2 l_{2}}{2}}\left(b_{1}\left(\xi_{1}^{2}-\gamma_{2}^{2}\right)+2 b_{2} \gamma_{2} \xi_{1}-i b_{3}\left(\xi_{1}^{2}+\gamma_{2}^{2}\right)\right)^{l_{2}} \gamma_{2}^{n-2 l_{2}}$,

which is not possible (note that $b_{1} b_{2} b_{3} \neq 0$ ). This concludes the proof of the lemma.

Lemma 4. Let $l_{1}=l_{2}$ and $f_{j}$ be a polynomial in the variable $\gamma_{2}$ and a rational function with respect to the variable $\xi_{1}$. Then condition $\mathcal{A}\left[f_{j}\right]=0$ implies

$$
f_{j}= \begin{cases}0, & \text { for } \quad j \in \mathbb{Z} \backslash\{0\}, \\ \text { constant }, & \text { for } j=0 .\end{cases}
$$

Proof. Let $l_{1}=l_{2}$ and $j \in \mathbb{Z} \backslash\{0\}$. From $\mathcal{A}\left[f_{j}\right]=0$ and after simplifying by $l_{2}\left(\xi_{1}-i \gamma_{2}\right)$ we have that

$$
\mathcal{S}\left[\left(\xi_{1}+i \gamma_{2}\right) \frac{\partial f_{j}}{\partial \gamma_{2}}+2 i \xi_{1} \frac{\partial f_{j}}{\partial \xi_{1}}\right]+2 j \xi_{1}\left(\left(i b_{2}-b_{1}\right)\left(\xi_{1}+i \gamma_{2}\right)+i b_{3}\left(\xi_{1}-i \gamma_{2}\right)\right) f_{j}=0 .
$$

Solving it we get

$$
\begin{gathered}
f_{j}=K_{j}\left(\frac{x_{2}+i x_{1}}{\sqrt{x_{1}}}\right) e^{-i j \tan ^{-1}\left(\frac{-2 b_{2} \xi_{1}^{2}+b_{3}\left(\xi_{1}-i \gamma_{2}\right)^{2}+2 b_{1} \xi_{1}\left(\gamma_{2}+i \xi_{1}\right)}{2\left(b_{2}-b_{3}\right) \xi_{1}\left(\left(i \xi_{1}+\gamma_{2}\right)+b_{1}\left(3 \xi_{1}^{2}-2 i \xi_{1} \gamma_{2}-\gamma_{2}^{2}\right)\right)}\right)} \\
\frac{\left.\left(5 b_{1}+4 i b_{2}-3 i b_{3}\right) \xi_{1}^{2}+2\left(-2 i b_{1}+b_{2}-2 b_{3}\right) \xi_{1} \gamma_{2}-\left(b_{1}-i b_{3}\right) \gamma_{2}^{2}\right)}{\xi_{1}^{2}\left(\left(b_{1}-i b_{3}\right) \xi_{1}^{2}+2 b_{2} \xi_{1} \gamma_{2}-\left(b_{1}+i b_{3}\right) \gamma_{2}^{2}\right)^{j / 2}} .
\end{gathered}
$$

Since $f_{j}$ must be a polynomial in the variable $\gamma_{2}$ and a rational function with respect to the variable $\xi_{1}$ and $b_{1} b_{2} b_{3} \neq 0$ we must have $f_{j}=0$. Now assume $j=0$. From $\mathcal{A}\left[f_{0}\right]=0$ after simplifying by $l_{2} \mathcal{S}\left(\xi_{1}-i \gamma_{2}\right)$, we have that

$$
\left(\xi_{1}+i \gamma_{2}\right) \frac{\partial f_{0}}{\partial \gamma_{2}}+2 i \xi_{1} \frac{\partial f_{0}}{\partial \xi_{1}}=0 .
$$

Solving it we get

$$
f_{0}=K_{0}\left(\frac{\left(\gamma_{2}+i \xi_{1}\right)^{2}}{\xi_{1}}\right)
$$

where $K_{j}$ is any smooth function. Since $f_{0}$ must be a polynomial in the variable $\gamma_{2}$ and a rational function with respect to the variable $\xi_{1}$ we must have that $K_{0}$ is a polynomial. Proceeding as in the proof of Lemma 3 we get that either $f_{0}$ is constant or

$$
\left(\gamma_{2}+i \xi_{1}\right)^{n}=\sum_{l_{2}=0}^{n / 2} \bar{G}_{l_{2}, l_{2}, \frac{n-2 l_{2}}{2}}\left(b_{1}\left(\xi_{1}^{2}-\gamma_{2}^{2}\right)+2 b_{2} \gamma_{2} \xi_{1}-i b_{3}\left(\xi_{1}^{2}+\gamma_{2}^{2}\right)\right)^{l_{2}} \gamma_{2}^{n-2 l_{2}} .
$$


But this last case is not possible and thus $f_{0}$ is constant. This concludes the proof of the lemma.

Proof of Theorem 1. We first show by backwards induction that $G_{j}=0$ for $j=-n, \ldots,-1$. For $j=-n$ we need to solve $\mathcal{A}\left[G_{-n}\right]=0$, and it follows from Lemmas 3 and 4 that $G_{-n}=0$. Then using that $\mathcal{B}[0]=0$ by induction we obtain that for any $j=-n, \ldots,-1$, the coefficient of $\Omega_{2}^{j}$ in (12) satisfies that $\mathcal{A}\left[G_{j}\right]=0$. Then the same arguments that we used for $G_{-n}$ imply that $G_{j}=0$ for $j=-n, \ldots,-1$. Moreover, $G_{0}$ satisfies that $\mathcal{A}\left[G_{0}\right]=0$ and again by Lemmas 3 and 4 we get that $G_{0}$ is a constant. Since we can always assume that $G$ has no constant term, then $G_{0}=0$. Note that $\mathcal{B}[0]=0$ and by induction we obtain that for any $j=1, \ldots, n$, the coefficient of $\Omega_{2}^{j}$ in (12) satisfies $\mathcal{A}\left[G_{j}\right]=0$. Again by Lemmas 3 and 4 , we get $G_{j}=0$ for $j=1, \ldots, n$. This completes the proof of the theorem.

\section{ACKNowledgment}

The authors would like to thank Prof. Andrzej Maciejewski for pointing out the remaining case in the integrability of the Suslov problem.

\section{REFERENCES}

[1] Y.N. Fedorov, A.J. Maciejewski, and M. Przybylska, The Poisson equations in the nonholonomic Suslov problem: integrability, meromorphic and hypergeometric solutions, Nonlinearity 22 (2009), no. 9, 22312259 .

[2] E. I. Kharlamova-Zabelina, Rapid rotation of a solid body about a fixed point with non-holonomic constraints, Vestnik Moskov. Univ. Ser. Mat. Meh. Astr. Fiz. Him. 12 (1957), 25-34.

[3] V.V. Kozlov, Symmetries, topology and resonances in Hamiltonian mechanics, Results in Mathematics and Related Areas (3), vol. 31, Springer-Verlag, Berlin, 1996.

[4] A. J. Maciejewski and M. Przybylska, Non-integrability of the Suslov problem, Regul. Chaotic Dyn. 7 (2002), no. 1, 73-80.

[5] Andrzej J. Maciejewski and Maria Przybylska, Nonintegrability of the Suslov problem, J. Math. Phys. 45 (2004), no. 3, 1065-1078.

[6] G.K. Suslov, Theoretical mechanics., Gostehizdat, vol. 107.

[7] S. L. Ziglin, On the absence of a real-analytic first integral in some problems of dynamics, Funktsional. Anal. i Prilozhen. 31 (1997), no. 1, 3-11, 95.

[8] S.L. Ziglin, On the absence of an additional first integral in the special case of the G. K. Suslov problem, Uspekhi Mat. Nauk 52 (1997), no. 2(314), 167-168.

${ }^{1}$ North Carolina State University, Raleigh, NC, 27695-8205, USA

E-mail address: amahdi@ncsu.edu

${ }^{1}$ Faculty of Applied Mathematics, AGH University of Science and Technology, Al. MickIEWICZA 30, 30-059 Kraków, POLAND

22Departamento de Matemática, Instituto Superior Técnico, 1049-001 Lisboa, Portugal

E-mail address: cvalls@math.ist.utl.pt 